Voix et Images

volxetimages

\title{
Saint-Denys Garneau ou le procès métonymique
}

\section{Noël Audet}

Volume 1, numéro 3, avril 1976

\section{Gérard Bessette}

URI : https://id.erudit.org/iderudit/200042ar

DOI : https://doi.org/10.7202/200042ar

Aller au sommaire du numéro

Éditeur(s)

Les Presses de l'Université du Québec

ISSN

0318-9201 (imprimé)

1705-933X (numérique)

Découvrir la revue

Citer cet article

Audet, N. (1976). Saint-Denys Garneau ou le procès métonymique. Voix et Images, 1(3), 432-441. https://doi.org/10.7202/200042ar d'utilisation que vous pouvez consulter en ligne.

https://apropos.erudit.org/fr/usagers/politique-dutilisation/ 


\section{Saint-Denys Garneau ou le procès métonymique ${ }^{1}$}

Depuis la célèbre étude de Roman Jakobson consacrée à deux types d'aphasie ${ }^{2}$, étude qui mettait en lumière les deux axes du langage, on connaît mieux le fonctionnement métonymique et métaphorique du langage poétique ou de la prose. Le bien-fondé de cette théorie a encore été corroboré il y a peu de temps par des faits cliniques, comme le rapporte luimême Jakobson dans un article intitulé "les Règles des dégâts grammaticaux ${ }^{3}$ ".

Résumons en quelques lignes les principaux éléments de cette théorie. Le discours se développe selon deux procédures sémantiques distinctes, deux "modes d'arrangement" ou deux axes. Le premier est celui de la combinaison: c'est-à-dire l'axe syntagmatique où les unités linguistiques simples se combinent en unités plus complexes pour produire une signification. Aspect horizontal, linéaire du signifiant, on l'appellera également l'axe de la contiguïté, ce qui en dernier ressort fonde le processus métonymique, la métonymie étant définie comme un rapport de contiguïté spatio-temporelle.

Le second axe est celui de la sélection (de la concurrence des termes). ou l'axe paradigmatique, c'est-à-dire celui du répertoire des termes similaires possibles (des synonymes aux antonymes). Axe de la similarité donc, qui fonde le processus métaphorique. La métaphore consisterait en un bond paradigmatique d'un terme à un autre "pris" dans la même échelle d'équivalences.

Dans un autre chapitre des Essais, Jakobson définit la fonction poétique en faisant précisément intervenir les deux axes qui entrent dans une sorte d'interférence:

La fonction poétique projette le principe d'équivalence de l'axe de la sélection sur l'axe de la combinaison ${ }^{4}$.

Or plusieurs chercheurs se sont autorisés de cette phrase, à tort nous semble-t-il, pour en conclure que la prose avait un fonctionnement presque exclusivement métonymique alors que la poésie se ramenait pratiquement toute à sa fonction métaphorique. De même, quand Jakobson explique "la primauté du procédé métaphorique dans les écoles romantiques et 
symbolistes [... et] la prédominance de la métonymie qui gouverne et définit effectivement le courant littéraire qu'on appelle "réaliste 5 ", on a vite fait de passer aux équations suivantes: romantisme/symbolisme = poésie: réalisme $=$ prose. Dans cette optique, on en arriverait à dire que c'est la quantité relative de métaphores qui détermine la "qualité " poétique. L'idée n'est pas sans fondement, puisque Jakobson lui-même la pose et la nuance (la nie) dans le même paragraphe:

La prose, au contraire, se meut essentiellement dans les rapports de contiguïté. De sorte que la métaphore pour la poésie et la métonymie pour la prose constituent la ligne de moindre résistance, ce qui explique que les recherches sur les tropes poétiques soient orientées principalement vers la métaphore. La structure bipolaire effective a été artificiellement remplacée, dans ces recherches, par un schème unipolaire amputé... 6 .

En conséquence, si l'on fait de la prépondérance des métaphores le principe d'identification de la poésie, une analyse linguistique de l'œurre de Saint-Denys Garneau, où la prédominance du procès métonymique saute aux yeux, nous force à conclure qu'il n'est qu'un prosateur égaré dans le vers libre. Ou bien, si l'on croit malgré tout à son statut de poète, il faut convenir que la question est mal posée et reviser nos prémisses. C'est cette dernière attitude que j'adopterai.

II m'apparaît nécessaire en effet de proposer l'hypothèse suivante: s'il est juste que la fonction poétique déplie le paradigme en syntagme, ne faudrait-il pas ajouter, comme je vais tenter de le montrer: de la même manière, la fonction poétique transforme les syntagmes combinés selon le procès métonymique en équivalences paradigmatiques, c'est-à-dire, en d'autres termes, que l'axe de la combinaison est reversé sur l'axe de la sélection. Cette hypothèse, si elle se vérifiait, pourrait avoir des conséquences pour la définition du langage poétique, dont l'essence ne consisterait plus en une quantité de figures. ni en la projection d'un axe sur l'autre, mais bien plutôt en la présence simultanée des deux axes et surtout en leur réversibilité ou reconversion permanente. Au lieu que seul le paradigme se déplie en syntagmes, on aurait aussi des syntagmes se condensant, se repliant en paradigmes.

\section{UN RECOURS QUASI EXCLUSIF AUX RAPPORTS DE CONTIGUÏTÉ}

Relisons des poèmes comme "le Jeu", "Saules", "Cage d'oiseau", "Accompagnement", "Silence», etc.,. en parallèle avec le chapitre de Jakobson intitulé "le Trouble de la similarité ${ }^{7}$, et l'on sera frappé par la prédominance très nette des opérations de contiguïté et le refoulement relatif de la similarité dans l'œuvre poétique de Saint-Denys Garneau (il va de soi que nous nous situons ici dans la normalité linguistique et non dans l'aphasie). Jakobson, citant Goldstein, constate que "les malades de ce type "saisissent les mots dans leur signification littérale mais ne parviennent pas à comprendre le caractère métaphorique de ces mêmes mots $^{8}$. Ainsi, il donne l'exemple de celui qui ne se souvient pas du mot 
"boussole" mais peut très bien en faire la description métonymique selon les parties et l'utilisation qu'on en tire: direction, aiguille aimantée, indique le Nord...

De même, quand la contiguïté domine, les structures syntaxiques sont très fortes et apparentes. Cette charpente de la phrase est la dernière à disparaître dans l'aphasie; en d'autres termes, la structure de la phrase peut demeurer intacte mais elle est impuissante à véhiculer le sens, parce qu'elle n'est plus nourrie par l'axe paradigmatique. Que l'on songe aux nombreuses relatives chez Saint-Denys Garneau, aux concessives, conditionnelles, circonstancielles de lieu, de temps, aux conjonctions "et", "mais "... qui indiquent la prépondérance qu'accorde l'auteur à l'axe de la contiguïté. Ce choix révèle bien sûr un style particulier, mais nous verrons qu'il se trouve lié à la thématique elle-même et en constitue peutêtre la justification.

Soit le poème "Cage d'oiseau " :

1 Je suis une cage d'oiseau Une cage d'os

Avec un oiseau

II L'oiseau dans sa cage d'os

C'est la mort qui fait son nid

III Lorsque rien n'arrive

On entend froisser ses ailes

IV Et quand on a ri beaucoup

Si l'on cesse tout à coup

On l'entend qui roucoule

Au fond

Comme un grelot

$\checkmark \quad$ C'est un oiseau tenu captif

La mort dans ma cage d'os

VI Voudrait-il pas s'envoler

Est-ce vous qui le retiendrez

Est-ce moi

Qu'est-ce que c'est

VII II ne pourra s'en aller

Qu'après avoir tout mangé

Mon cœur

La source du sang

Avec la vie dedans

VIII Il aura mon âme au bec ${ }^{9}$.

Le premier vers constitue une. métaphore, cependant cette métaphore n'est qu'un leurre, un premier piège dans l'élaboration du sens. En effet, Je: cage d'oiseau, où "oiseau" devrait, métaphoriquement, libérer les sèmes de joie, chant, poésie. Or la suite du poème, par le développe- 
ment de la contiguïté spatiale (engendrement d'une série de rapports métonymiques), se charge de nier ce premier faux sens, soit celui de la métaphore. Dès le deuxième vers en effet, "une cage d'os" souligne la défaite de la métaphore "oiseau» avec ses connotations habituelles. En fait, le mot oiseau du premier vers était pris au degré zéro, n'indiquant pas du tout une présence mais simplement la nature de la cage (cage d'oiseau par opposition à cage de fauve); et le poète poursuit en sentant le besoin de préciser la matière de la cage (rapport métonymique): cage d'os, dont les connotations sont antithétiques par rapport à celles de "oiseau". Il continue en établissant un nouveau rapport métonymique de contiguité spatiale indéfinie: "avec un oiseau", la seule précision consistant à affirmer une présence, sans expliciter sa localisation (dans la cage, à côté, au-dessus ?). De même remarquons l'article indéfini de “ un oiseau ".

Poursuivons la lecture, strophe II:

$L$ 'oiseau dans sa cage d'os

$C^{\prime}$ 'est la mort qui fait son nid

Un article défini et un possessif: on est donc passé du degré zéro de "oiseau" à sa détermination par l'indéfini, puis à sa détermination précise par l'article défini suivi d'une circonstance de lieu, enfin le tout étant ramassé dans une équivalence paradigmatique, c'est-à-dire une définition exacte: l'oiseau égale la mort qui fait son nid.

Le sens se constitue en glissant de syntagme en syntagme, en culbutant le long d'une linéarité dont chaque portion n'est pas équivalente, c'est-à-dire que d'un syntagme à l'autre il y a procès du sens, avancée de la signification, d'une façon discrète, au ras des mots - par bourgeonnement pourrait-on dire, où règne en maître la contiguïté sémantique et positionnelle. C'est la transformation progressive d'un syntagme par addition ou retrait d'éléments syntaxiques.

C'est ainsi que par précision de tous les rapports métonymiques, Saint-Denys Garneau arrive à détruire une métaphore-leurre et à établir la véritable métaphore du poème: l'oiseau = la mort, soit la négativité absolue et active ("qui fait son nid").

Le reste du poème ne sera que le développement métonymique de ces deux aspects de la mort, négativité et activité captive. C'est pourquoi, par exemple, le premier vers "Je suis une cage d'oiseau" reprend son seul sens possible dans le contexte: le poète devient objet inerte, impuissant, inactif, il est l'enveloppe contenant (rapport de contiguïté) la mort-oiseau active. Il y a plus, le poème se développe selon une suite de synecdoques de l'oiseau: ses ailes, sa voix (roucoule), sa nourriture (le cœur...), son bec.

La cinquième strophe, encore par une simple transformation syntaxique - le changement de "sa" en "ma" - permet la soudure de toutes les significations du poème en posant une métaphore (mort: oiseau captif) sur un rapport métonymique qui la détruit: «dans ma cage d'os" où le possessif rapporté au locuteur marque une relation de propriété qui trans- 
forme le sens de "cage d'os", devenue synonyme de squelette ou simplement de corps appelé à mourir; en conséquence, l'oiseau n'y trouve plus de lieu, son seul lieu étant dans la métaphore d'une cage (d'oiseau). En fait, Saint-Denys Garneau se perçoit lui-même selon le procès métonymique, c'est-à-dire qu'il décrit le réseau de relations entre son' corps, son cœur, son âme et l'univers non pas au moyen de la rupture métaphorique mais dans la plus sévère contiguïté. Le progrès qu'amorce la signification dans cette cinquième strophe induit déjà le vers final, où la partie non mortelle (non proie de l'oiseau), l'âme, pourra fuir, mais liée encore à l'oiseaumort selon un rapport de cause à effet, traduit au niveau de la syntaxe dans une relation synecdochique : "Il aura mon âme au bec."

En terminant cette brève analyse, j'attire l'attention sur la strophe VII dans laquelle l'auteur écrit: "Il ne pourra s'en aller/Qu'après avoir tout mangé/Mon cceur/La source du sang/Avec la vie dedans". C'est le procès métonymique en clair. Saint-Denys Garneau aurait pu dire: mon ccur, source du sang, vie... et nous aurions eu affaire à des métaphores, soit des équivalences paradigmatiques projetées sur l'axe syntagmatique. Au contraire, il a choisi le versant de la contiguïté pour poser les rapports entre "tout" et le cœur et "la source du sang" (une fonction du cœur), «avec la vie dedans" (ce qui représente encore le cœur mais vu comme principe de vie, et où la préposition avec marque bien le refus de la métaphore et le soulignement de la contiguïté: ce qui accompagne, ce qui est juxtaposé à...).

C'est une véritable vision métonymique du monde, d'un monde fragmenté, éparpillé en autant de syntagmes disjoints/conjoints dont le travail consiste à produire une métaphore, c'est-à-dire à retransformer le texte en paradigme. Car la signification globale du poème est bien métaphoriaue (la mort = oiseau captif), mais elle s'est presque entièrement construite en prenant appui sur les rapports de contiguïté. C'est pourquoi j'affirme qu'en poésie, du moins ici, le procès métonymique est reversé le long de l'axe paradigmatique, et c'est après coup seulement que l'on est en mesure de lire les équivalences: elles sont le résultat du travail de l'écriture métonymique et non l'inverse, comme on le verra chez Alain Grandbois.

Une lecture des équivalences paradigmatiques dégagées:

JE $=$ CAGE D'OISEAU

OISEAU = négativité / captivité / activité

$\begin{array}{lll}\text { (mort } & \text { captif } & \text { fait son nid } \\ \text { os } & \text { cage } & \text { froisser ses ailes } \\ \text { rien } & \text { grelot } & \text { roucoule } \\ \text { n'arrive } & \text { (strophes } & \text { (grelot) } \\ \text { on a ri } & \text { VI et VII) } & \text { s'envoler } \\ \text { on cesse) } & & \text { tout mangé }\end{array}$

Transformation finale: rupture de la cage, libération liée:

$\mathrm{JE}=\hat{\mathrm{A} M E}$ à son bec 


\section{LA CONTIGUITTÉ : LIEU D'UN DRAME (THÉMATIQUE)}

Chez Saint-Denys Garneau, la contiguïté est si forte et si présente qu'elle fait l'objet d'une thématique centrale dans l'œuvre. Dans "Accompagnement", poème bien connu, je me contenterai de relever quelques termes du lexique et quelques syntagmes. Le tout parle de lui-même.

“Je marche à côté d'une joie ${ }^{10}$ ". Ici, la thématique est nettement inscrite dans un rapport de contiguïté marqué par une locution syntaxique: à côté de. Cette contiguité qui ne peut pas devenir paradigme ou équivalence, c'est tout le drame de Saint-Denys Garneau, sa thématique fondamentale, et je fais l'hypothèse que la prédominance du procès métonymique (axe de la contiguïté) est liée à la thématique elle-même.

“D'une joie à moi que je ne puis pas prendre». C'est dire encore la proximité de l'objet, dans une relation de propriété, mais en même temps l'impossibilité de s'en saisir, soit l'impossibilité de changer le syntagme en paradigme.

«Je marche à côté de moi en joie». Le poète est amené à produire un double de lui-même, dans une véritable opération schizophrénique, double de lui-même, c'est-à-dire équivalence paradigmatique glissée du discours qui est incapable de l'assumer à l'univers imaginaire/réel du poète. Le syntagme s'est inventé un double, mais il doit se contenter "pour le moment de cette compagnie", parce qu'il n'a pas encore trouvé le moyen de se transposer dans l'axe paradigmatique, où l'équivalence l'exempterait de cet accompagnement. La suite du poème ne pouvait que proposer une suite d'opérations "prises" ou gelées dans cette contiguïté : échanges, opérations, alchimies, transfusions, déménagements d'atomes, jeux d'équilibre. (Jeux d'équilibre: jeux de mots, équivalences; on connaît l'importance de ce sous-thème de l'équilibre dans l'œuvre).

Et le poète trouve la solution, quand il sera "transposé " (quatrième strophe). Le mot est bien de lui, transposé: posé ailleurs, dans un autre ton, un autre domaine, un autre axe, c'est-à-dire posé en équivalence à lui-même, dans le paradigme de l'être et non plus dans le syntagme du non-avoir.

À ce sujet, j'aimerais introduire ici une autre hypothèse, soit celle qui définirait l'être et l'avoir comme appartenant à deux axes différents, l'être étant paradigmatique et l'avoir syntagmatique. En effet, l'être ne se mesure pas, il n'est pas quantifiable, il ne peut que se définir, c'est-à-dire se projeter dans des équivalences paradigmatiques, se déplier en similarités. Monsieur $X$ est poète, malade du cœur, brillant, de taille moyenne... Alors que l'avoir se situe essentiellement le long de l'axe syntagmatique; s'approprier quelque chose c'est ajouter à côté, autour de soi, ou établir une relation de contiguïté: il possède une maison, qui abrite ses deux chats (le possessif), qui... etc.

En conséquence, je dirais que Saint-Denys Garneau travaille dans l'axe de la contiguïté et pose tout en termes syntagmatiques parce qu'il se sent dépossédé de tout, y compris et surtout de sa propre existence, 
ce qui le maintient malgré lui dans l'axe de l'avoir. Ce sentiment de nonêtre le pousse à vouloir continuellement s'approprier les choses par le discours métonymique, la poésie seule arrivant à opérer la transmutation de l'axe de l'avoir en être, précisément en reversant, comme je le soulignais, la série de syntagmes le long de l'axe paradigmatique (la suite de métonymies travaille à produire une métaphore, comme les avoirs chercheraient à produire une métamorphose).

\section{COMMENT LA CONTIGUİTÉ DEVIENT ÉLÉMENT D'UN ART POÉTIQUE}

De style particulier à vision du monde qu'elle était, la contiguïté devient, dans "Un poème a mijoté... ${ }^{11}$ », un procédé qui situe le "faire" poétique tout en marquant ses limites. Le poème s'écrit dans et contre le langage, soit malgiré l'impossibilité habituelle du syntagme à se re-projeter en paradigme.

“Un poème a mijoté tout le jour» - “mijoté» définit bien la situation d'un corps à la frontière même de deux états, liquide et gazeux, sans qu'il $y$ ait jamais rupture, soit passage d'un état à l'autre. C'est le mauvais équilibre, le point zéro, lieu où la contiguïté se prenant pour objet fige sa relation: il n'y a de voie ni d'un côté ni de l'autre par rapport à la frontière de l'équilibre. Mijoté, le poème est source qui n'arrive pas à sourdre:

On a senti sa présence tout le jour soulevante

Comme une eau qui gonfle

Et cherche une issue

Mais cela s'est perdu dans la terre

II n'y a plus rien ${ }^{12}$.

C'est bien la signifiance qui mijote, pulsions, rythmes, fragments d'image, murmure qui cherche son point de condensation pour jaillir mais qui n'y arrive pas, pour aller finalement se reperdre dans la terre, ou l'informe et l'innommé.

Saint-Denys Garneau commence son poème par l'article indéfini, et l'on en comprend la signification: le récit raconte que le poème ne viendra pas, donc il doit demeurer sémantiquement indéfini. Là où il y a surprise et, je crois, "contamination" par contiguïté, c'est lorsque l'auteur écrit, au troisième vers: “On a senti..." Qui est ce "on" sinon le “je” du locuteur se transformant en pronom indéfini pour marquer encore la défection du poème dans la conscience du poète et, par relation métonymique, la défection de la conscience elle-même qui n'arrive pas à le penser.

La contagion par contiguïté est même plus nette dans la deuxième partie du poème où, suite à une comparaison ("comme des fous"), le discours passe du "on", pronom indéfini mis d'abord pour «je", à un «on» pluriel mis pour nous, et enfin par une nouvelle transformation syntaxique, à un “ils" qui suit un retour de la même comparaison :

On a marché tout le jour comme des fous 
Comme des fous tout à coup attentifs

À un démêlement qui se fait dans leur cerveau

Comme s'ils allaient retrouver

$$
[\ldots]
$$

Autant dire que le poème qui essayait de naître d'un «je» in-défini (d'un jeu non défini entre paradigme et syntagme, d'un «je» qui se nie en tant qu'être) se trouve progressivement coupé de sa relation à l'auteur et est pris en charge par un "ils" (des fous) qui n'assumera plus qu'une pure narrativité, c'est-à-dire l'histoire de la non-naissance du poème.

Le poème est en deçà de, près de, implosion, proximité pure, il n'arrive pas à naître parce que le "démêlement" ne se fait pas. On passe en effet de "mijoté" à un "démêlement" qui ne peut pas s'effectuer, autre terme indiquant fortement l'axe de la contiguïté, l'enchaîné dans les deux sens de: lié à, et empêché de.

Le poème est là, bien sûr, comme en fait foi l'œuvre de Saint-Denys Garneau, mais l'histoire de sa naissance s'écrit comme celle d'un avortement, qui s'agrandira aux dimensions cosmiques: "Annihile le monde entier $»$.

Nous avons assisté à la contagion de l'indéfini par les transformations suivantes: UN $\rightarrow$ ON (pronom indéfini mis pour je) $\rightarrow$ ON (mis pour nous/ fous) $\rightarrow$ ILS (eux, objet) $\rightarrow$ NÉANT (cosmos aboli, comme le poème dans l'esprit). Contagion, comme dans le phénomène de capillarité, de proche en proche, sans rupture spatiale ni bond paradigmatique, si ce n'est un seul: soit le passage du poème, univers du discours, à l'univers matériel. Ce saut paradigmatique se fait bel et bien selon le procès métaphorique, c'est-à-dire dans l'axe de la similarité. En effet, Saint-Denys Garneau décrit le poète qui s'affole devant la lenteur du poème à naître ( " tout le jour"), au point d'en perdre son identité, comme les fous "s'affolent de la lenteur du jour à naître" - et cette équivalence est rendue possible grâce à la médiation d'une image qui se trouve au milieu du poème: démêlement dans leur cerveau =lumière (sens) $=$ lumière du jour; et implicitement nous pouvons constater: démêlement $=$ poème $=$ cosmos. La poésie est donc cosmogonie, et on comprend mieux la fin du poème qui proclame que le monde se trouve annihilé parce que le poème n'est pas venu: le monde retourne à la confusion, à l'innommé, en dehors du sens.

On voit de nouveau un poème, presque entièrement construit selon le procès métonymique, prendre tout à coup une signification métaphorique et même plusieurs ${ }^{13}$. C'est que l'écriture poétique, même opérée au ras de la contiguïté, construit une signification qui s'ouvre et se retransforme en paradigme, la censure du poète n'y pouvant rien.

\section{ÉCRITURE ET CENSURE}

Ces dernières constatations m'amèneraient à trouver une autre motivation, d'ordre psychanalytique celle-là, à l'écriture syntagmatique de SaintDenys Garneau. La syntaxe en effet est l'instrument privilégié de la censure. 
par le rétrécissement ou la clôture du sens. «Quel pivot, j'entends, dans ces contrastes, à l'intelligibilité ? il faut une garantie - La Syntaxe - 14." Saint-Denys Garneau serait bien le poète de la dépossession (dans l'axe syntagmatique), de la rareté, de la censure, ou peur du signifiant multiple, peur qui aboutit finalement au silence, soit à la censure totale.

Silence

Toutes paroles me deviennent intérieures

Et ma bouche se ferme comme un coffre

qui contient des trésors

Et ne prononce plus ces paroles dans le temps,

Mais se ferme et garde comme un trésor, des paroles en passage, ses paroles

Hors l'atteinte du temps salissant, du temps passager ${ }^{15}$.

On aura remarqué, encore ici, le même développement par transformations syntaxiques successives d'un segment de parole, ou le déplacement d'une même pulsion. Nous observons toujours la prédominance de l'axe de la contiguïté; nous le retrouverions dans "C'est eux qui m'ont tué " ( $p .201)$, etc. Et Julia Kristeva fait remarquer à juste titre:

On peut aujourd'hui préciser, en écoutant le discours des analysants à partir de Freud et de ce que la linguistique structurale a pu articuler du fonctionnement linguistique, que la condensation (la métaphore) résulte de la surdétermination, tandis que le déplacement (la métonymie) résulte d'une censure ${ }^{16}$.

Chez Saint-Denys Garneau, la censure chrétienne, entre autres, n'aurait-elle pas été intériorisée au point de transformer jusqu'au fonctionnement de son langage? Et je suis porté à considérer comme un produit de ce langage, impuissant à nommer l'être mais nommant cette impuissance même, le fait que la critique y ait toujours lu une profondeur métaphysique, une recherche angoissée de l'être ou de l'Être; ce qui se réduirait, peut-être un peu cavalièrement, à un problème de censure et à un fonctionnement particulier du langage.

Une remarque en terminant: le syntagmatique recoupe également toute distribution de masses dans l'espace. On s'étonnera moins de retrouver autant de tableaux dans l'œuvre de Saint-Denys Garneau. Le fait qu'il ait étudié la peinture n'explique pas grand'chose. J'y vois toujours la prépondérance manifeste de l'axe de la contiguité et le refoulement relatif de l'inquiétant saut paradigmatique où la parole s'ouvre, comme on s'ouvre les veines, c'est-à-dire sur des métaphores que ne peuvent plus rattraper la syntaxe ni la censure.

Noël Audet

1. La seconde partie de cet essai sera consacrée à Alain Grandbois et au procès métaphorique.

2. "Deux aspects du langage et deux types d'aphasie", dans Essais de linguistique générale, Paris, Éditions de Minuit, 1970. 
3. Dans Langue, discours, société, Paris, Seuil, 1975.

4. Essais de linguistique générale, p. 220.

5. Ibid., p. 62.

6. Ibid, p. 67.

7. Ibid., p. 49.

8. Ibid., p. $\mathbf{5 6 .}$

9. Poésies complètes, Montréal, Fides, 1949, p. 96-97.

10. C'est moi qui souligne.

11. Poésies complètes, p. 131.

12. Id.

13. J'ai peine à ne pas voir dans ce poème le symbole même de la pulsion libidinale refoulée, réprimée par le symbolique, en l'occurrence l'ordre social et la morale chrétienne. La figure de ce père symbolique y est incarnée par le soleil où "la lueur s'en re-va", "hors de vue", c'est-à-dire pour être cachée à la vue du fils, parce qu'il n'y a pas eu passation des pouvoirs. D'où également l'abolition de l'univers, objet du désir refoulé.

14. S. Mallarmé, CEuvres complètes, Paris, Gallimard, “La Pléiade», 1961, p. 385.

15. Poésies complètes, p. 122.

16. La Révolution du langage poétique, Paris, Seuil, 1974, p. 230. 